\title{
Object Sorting Robot Mechanism on the Basis of Colour, Height and Type (Metal or Non-metal) Using Human Voice Input
}

\author{
Dr. K Vijayan, Anjima S Nair, D Shruthi, Krishna Manoj, Rohan Sharma
}

\begin{abstract}
Automation is a very broad term which is basically used to handle different mechanisms and processes to reduce human efforts, time consumption and human energy. It reduces manual efforts, costs and is faster as well as more accurate than human beings. Sorting of objects is a very important aspect of agricultural machineries, food processing industries and various other industrial applications. Many kinds of intelligent robots are emerging these days. These systems collaborate with humans to accomplish tasks like sorting in an unstructured environment. Sorting plays a very significant role in the manufacturing and production industries. Also, sorting is one of the widely researched topics in today's time. This paper describes about a robotic mechanism for sorting objects. The Sorting system uses a TCS3200 Colour Sensor, an SR-04 Ultrasonic sensor, Metal detector, Bluetooth module and a Robotic push mechanism. Three different parameters like colour, height and type of object (metal or non-metal) is detected by setting the threshold and by giving the input one at a time by using human voice command via a Bluetooth module integrated with the Arduino. The three colours detected include red, blue and green. Also the range of distances or heights measured by the ultrasonic sensor reaches up to $200 \mathrm{~cm}$ The overall sorting is then carried out by the robotic mechanism.
\end{abstract}

Index Terms: Bluetooth Module, D C Motor, Metal Detector Sensor, Ultrasonic Sensor, TCS3200.

\section{INTRODUCTION}

The traditional robot programming required the programmers to mention every action to be performed by the robot in the code given to it. This method was time consuming and involved long codes to be written to do a particular task. The researchers across the globe, then decided to make Intelligent robots which are capable of making decisions on their own and acting upon it. The most common ways of communicating with robots, even today is human gestures, voice input and text based inputs. Various pick and place robot along with

Revised Manuscript Received on July 05, 2019.

K Vijayan, Department of Electronics and Communication, SRMIST, Chennai, India

Anjima S Nair, Department of Electronics and Communication, SRMIST, Chennai, India.

D Shruthi, Department of Electronics and Communication, SRMIST, Chennai, India.

Krishna Manoj, Department of Electronics and Communication, SRMIST, Chennai, India.

Rohan Sharma, Department of Electronics and Communication, SRMIST, Chennai, India other root mechanisms already exist wherein each part of a robotic arm is built and programmed to rotate and move

according to predefined angles. In this paper, we design a human interactive reasoning robot mechanism which sorts objects on the basis of parameters like colour, height and type (metal or non- metal) using human voice input via a Bluetooth module. This mechanism is used to sort objects on a particular confined space. The objects will be sorted based on the parameter given as input by the user at a particular time. When the user's desire is left unsatisfied or mismatched, the robot mechanism will respond with an error message displayed on the LCD screen attached to it indicating the aforementioned.

\section{LITERATURE SURVEY}

To reduce human errors in high end industrial processes, different types of robotic arm structures are used. These arms are quite expensive due to the material costs, the fabrication costs involved in the making of these arms, along with the programming required. Most of the robotic mechanisms are designed to reduce manual errors and perform jibs smoothly and efficiently. The paper prescribed in ref [2] infers that objects can be sorted by using a camera to capture the images of the objects followed by incorporating image processing technique to sort objects based on colour. Ref [3] infers that coloured objects can be detected using TCS3200 colour sensor which is a circuitry comprising of photodiodes and light to frequency converter mechanism. Also there are mechanisms wherein the shape of objects can be detected using MATLAB image recognition [1] or even by using the combination of an Image processing algorithm and classifier mechanism [4].

\section{EXISTING SYSTEM}

Traditionally, an industrial robot is dedicated to be used in a high-volume production industry using a specialized end effecter (gripper) which is constructed to retrieve the same part repeatedly from a known position and orientation. A robotic arm that operates with the help of DC servomotors are used to sort objects on the basis of any parameters like shape, size, colour or depending on the type of material. Usually, the objects are placed on a conveyer belt and the command to sort it according to the chosen parameter by the user is given manually through programs written on Arduino software which is most commonly used. For sorting objects based on colour, a colour sensor or image processing is used. For sorting objects based on weight, a load sensor is used which is usually attached beneath a conveyer belt on 
which different objects to be sorted are placed.Also for shape, image processing based on colour is used. The objects that satisfy the requirements are then picked and placed in a container using the robotic arm. This approach is ideal mainly for large-scale manufacturing operations. Unfortunately, this approach is not a good match for some industrial applications in which a large variety of part types need to be handled i.e. sorting numerous objects on the basis of various parameters. More flexible industrial robotics solutions have to be constructed and developed to handle such unstructured production applications. Also other traditional methods used for object sorting involve placing objects on a conveyer belt from which objects are sorted based on the input given to the system through the code itself and a robotic arm is also used which operates with the help of DC servomotors to sort objects on the basis of any parameter like shape, size, colour or the type of material. The arm is then used to pick the object from the conveyer belt to the bin kept nearby. Object sorting on the basis of colour usually involves either the colour sensor (TCS3200) or image processing techniques based on MATLAB software. At the same time, object sorting based on shape indirectly involves image processing on the basis of colour, while objects are sorted on the basis of weight using a load sensor. Generally, such systems tend to become very heavy due to weight of the conveyer belt along with the weight of the load sensor that is used to weigh the objects and other sensors and mechanisms used to support the system. Though this approach is ideal for very large scale industries, it is not so apt for small scale industries and industries that require the object to be sorted under various parameters, like in quality check for products. This need calls for more simple but efficient systems in the field of sorting.

\section{PROPOSED SYSTEM}

The proposed system uses a various parameter like colour sensor (TCS3200), an ultrasonic sensor, a metal detector and a Bluetooth module. Three different parameters like colour, shape i.e.., height and type of object is detected, the threshold for these parameters are set using a voice command from a distance Bluetooth module. The overall sorting is then carried out using the robot mechanism which moves on a confined space of area and uses a push mechanism and so the sorted objects are placed in a bin. Also, the proposed system focuses on sorting objects based on various parameters like colour, size and type of object by giving a voice input to the system which will specify the conditions based on which the objects need to be sorted. For sorting of objects based on colour, the TCS3200 colour sensor is used. For sorting objects based on size an ultrasonic sensor is used and for the type of the object, (metal or non-metal) a metal detector sensor is used. The voice input is given to this system using a Bluetooth module which connects the system to a smart phone which can be used to operate the system.

\section{BLOCK DIAGRAM}

The block diagram shown in Fig. 1 describes the entire system which is used to sort the objects or items according to their specifications. We are using multiple sensors like a colour sensor (TCS3200), an ultrasonic and a metal detector sensor to classify and sort the objects based on the input given to the robot mechanism. A Bluetooth device is used to give the human voice input based on the user's desired parameter to sort the objects. A threshold is set for the three parameters within which sorting can be accomplished for each parameter. Following this the object is sorted accordingly using a push piston mechanism which is activated by using a motor. The processor on the raspberry pi controls the servo motors which in turn are responsible for the movement of the robot mechanism. The relays are basically a switch responsible for controlling the Motor. When the required parameter is sensed by the robot mechanism, it will place the object on it and push it on a bin placed on the mechanism itself. An LCD screen placed on the robot mechanism itself will display the parameter specification on it, notifying the user of the successfully accomplished sorting or else an error message will be displayed to the user.

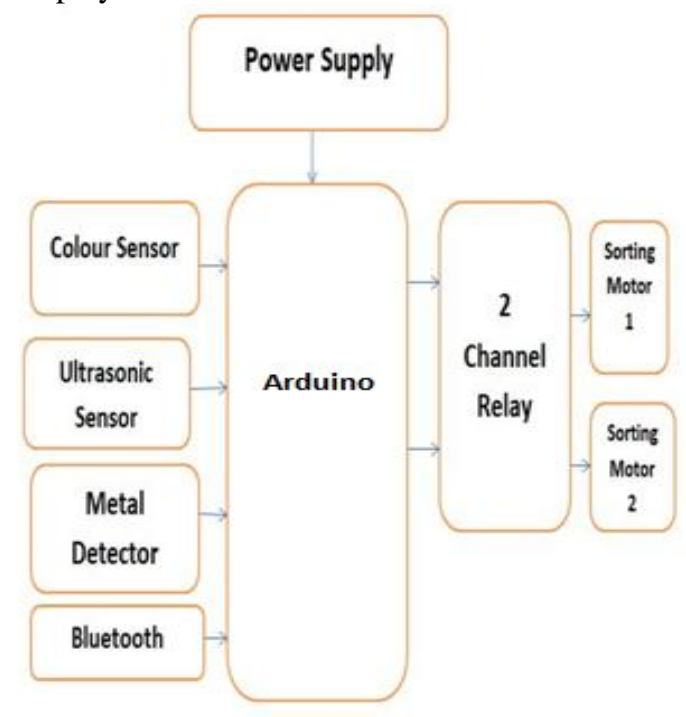

Fig. 1 : Block Diagram

\section{HARDWARE IMPLEMENTATION}

A. Arduino is basically an open-source platform most commonly serving the purpose of building electronics projects. It consists of a physical programmable circuit board which is the microcontroller along with the software, or IDE (Integrated Development Environment) used for programming. Using an Arduino allows the connection of a USB to it rather than using a separate code to run external information or hardware. Also the programming used in Arduino is usually embedded $\mathrm{C}$ which makes it user friendly and much simpler to understand and use. Different kinds of components can be used and attached to the Arduino board like the various sensors along with GPS (global positioning system),GSM modules (global system for mobile communication) etc. according to the requirements.

B. A switch that operates electrically is most often referred to as a relay. Relays are mostly based on the principle of electromagnetic theory even though solid state relays exist. A relay basically consists of a solenoid that is wounded by wire providing a low resistance path for magnetic flux along with a moving coil armature and a set of contacts. Whenever current pass through the circuit, the relay switches position and pass control from one switch to another.

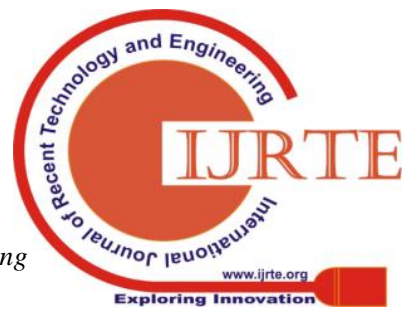


There never exists a proper connection between the two circuits as the link is between magnetic and mechanical. Relays are primarily used when a low power signal controls a circuit or when a single signal controls many circuits at a time.

C. An ultrasonic sensor is basically a combination of a transmitter, a receiver and a transceiver. The transmitter emits ultrasonic waves, while the receiver, receives the waves reflected back from the object. The time difference between the emitted and the reflected waves from the object is then calculated and converted into distance. In this project, an ultrasonic sensor is basically used to calculate the size of the object using its principle. It achieves an exceptional range of accuracy of height or distance and their corresponding fixed readings in a simple, compact and an easy to use packaging. It requires a power supply of 5 volts.

D. The TCS3200 senses coloured light with the help of an array of photodiodes present inside a circuitry consisting of photodiodes to detect the presence of light followed by a light to frequency or current to frequency converter. The Current-to-Frequency Converter basically converts the current signal from the photodiode to a square wave whose frequency is directly proportional to the applied light input intensity. Finally, by using the Arduino we can read the square wave output and the appropriate colour can be sensed and sorted. This is shown in Fig. 2.

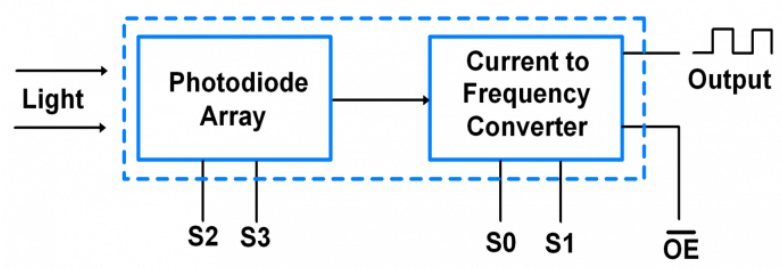

Fig. 2 : Principle of TCS3200

E. The Bluetooth module is commonly used for a serial port replacement to build a relation between a personal computer, laptop, mobile devices or other devices such technological systems and between the microcontroller of the robotic things etc. The Bluetooth module can be built up for baud rates from 1200 to $115200 \mathrm{bps}$.

F. Metal detector is basically an electronic component through which when electricity flows in and around the transmitter coil present inside the component, it leads to the creation and development of a magnetic field. If the detector is swept above a metal object, the magnetic field produced penetrates through the metal object and leads to the production of electric current in accordance with the electromagnetic theory leading to the detection of a metal object.

G. Any part of a system that controls the movement and mechanism of any other part of the system is called as an Actuator. A servomotor is an example of an actuator. It helps in changing the positions of different parts of the system very accurately and precisely in both angular and linear aspects. The input given to a servomotor is mostly an analog or digital signal which specifies the motion of a shaft. Servomotors are also said to show high performance than stepper motors. It basically works on the principle of Pulse Width Modulation.

H. More components are required like

- HDMI to VGA converter

- Power Cable

- LCD

- LED Lights

- Ribbon Cable

\section{SOFTWARE REQUIREMENTS}

Arduino IDE is an open source software that is used to write codes and running it and obtaining widespread outputs majorly used for student projects. It works on Windows, Mac and Linux. It is very easy to install and use.

\section{RESULTS AND DISCUSSION}

Firstly, for sensing the height, an ultrasonic sensor was used and three thresholds of $500 \mathrm{~cm}, 200 \mathrm{~cm}$ and $100 \mathrm{~cm}$ was set for objects classified as 'tall', 'medium' and 'small' respectively. An input was given by the user as 'small' using the Bluetooth module and the robotic mechanism moved on a confined space and carried out sorting. As shown in Fig. 3 and Fig. 4 screen an object with height $4.84 \mathrm{~cm}$ (which is under the range of 'small') was sensed and the same was displayed on the output console of Arduino. Also the user was notified of the successful sorting using an LCD screen.

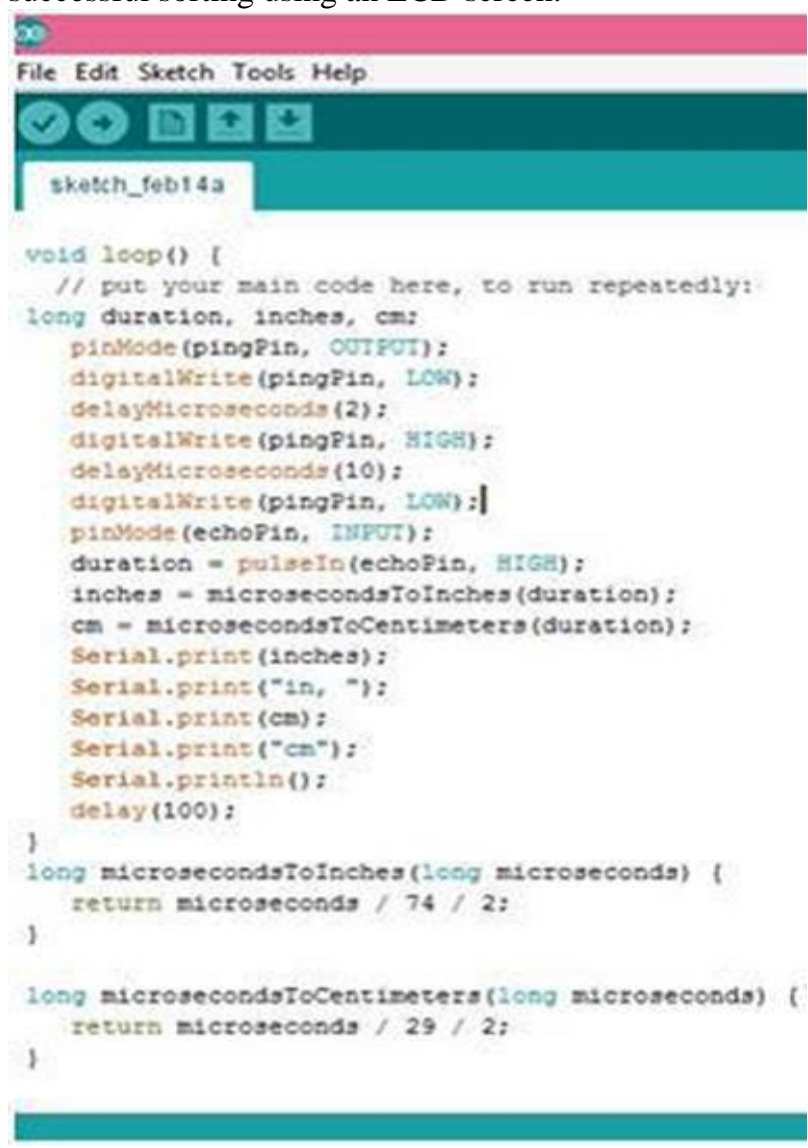

Fig.3: Snapshot of Arduino IDE 


\section{$\gg>$ \\ Distance Measurement In Progress Waiting For Sensor To Settle Distance: $4.84 \mathrm{~cm}$ $\gg>$}

Fig.4 : Distance Measurement

Secondly, for the colour parameter, TCS3200 was used. An input was given by the user as red through the Bluetooth module and the robot mechanism moved on the confined space on a predefined path and sensed the red coloured object. Also the user was notified of the successful sorting using an LCD screen attached to the robot mechanism.

Three colours red, blue and green were added and the results are shown below in Figures 5, 6 and 7 respectively.

\section{$\mathrm{R}=260 \quad \mathrm{G}=166 \quad \mathrm{~B}=165$ \\ $\mathrm{R}=260 \quad \mathrm{G}=166 \quad \mathrm{~B}=170$ \\ $\mathrm{R}=255 \quad \mathrm{G}=153 \quad \mathrm{~B}=153$ \\ $\mathrm{R}=260 \quad \mathrm{G}=175 \quad \mathrm{~B}=182$ \\ $\mathrm{R}=276 \quad \mathrm{G}=179 \quad \mathrm{~B}=176$ \\ $\mathrm{R}=265 \quad \mathrm{G}=166 \quad \mathrm{~B}=170$ \\ $\mathrm{R}=250 \quad \mathrm{G}=158 \quad \mathrm{~B}=159$ \\ $\mathrm{R}=255 \quad \mathrm{G}=166 \quad \mathrm{~B}=176$}

Fig.5 : Output of Red Color

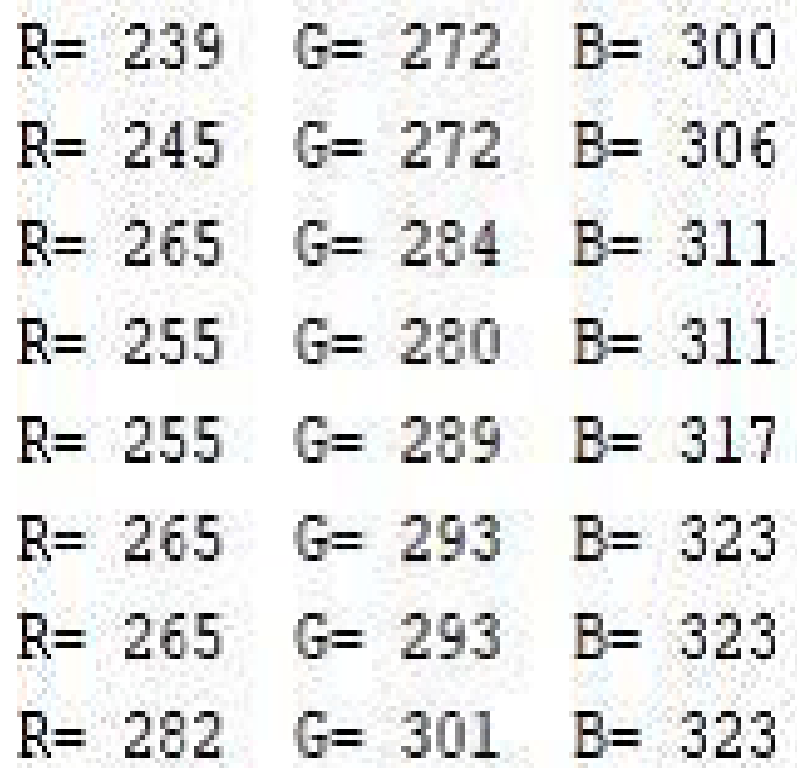

Fig.6 : Output of Blue Color

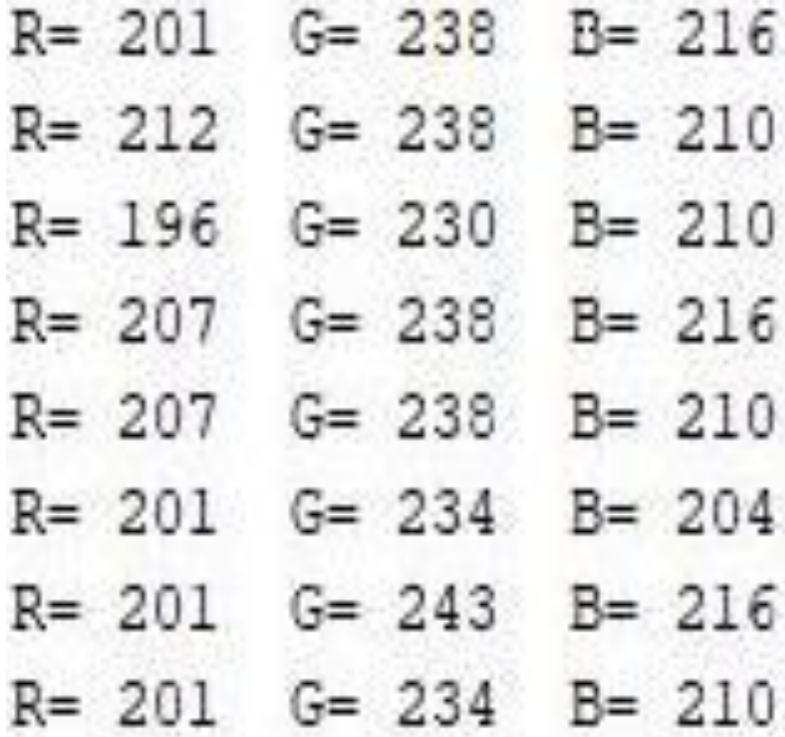

Fig. 7 : Output of Green Color

For the third parameter, the voice input was given by the user as either metal or non-metal and the robot mechanism sensed the same using a metal detector sensor followed by a notification displayed on the LCD corresponding to the successful sorting based on the type of the object.

The colour sensor TCS3200 gives a very firm response under various sunlight conditions. The response of the system is little slower than expected. To overcome these discrepancies, a recent version of the Arduino software, a better microcontroller or an advanced sensor can be used.

\section{CONCLUSION}

This project mainly focused to build an established, automated and functional robot mechanism which works with voice input given by using a Bluetooth module and sorts objects on the basis of three parameters individually at a time including colour, height and type of object (metal or non-metal) and the required output is obtained successfully. Hence, different types of objects based on the human input were successfully sorted by the robot mechanism which is set to move on a predefined confined space. The system focuses on an automated sorting mechanism, thereby reducing human error, time consumptions and cost.

\section{ACKNOWLEDGMENT}

We extend our sincere gratitude to the management of SRM Institute of Science and Technology for the tremendous opportunities that we received to strengthen our knowledge in the field of Electronics and Communication Engineering and to the Director of Engineering and Technology, The Head of the Department and to all our teachers for giving us the right instructions and guiding us in the right direction always.

\section{REFERENCES}

1. Priya Vinayak Garad, "Object sorting robot based on the shape", International Journal Of Advanced Research,Ideas and Innovations in Technology,2017,Volume 3, Issue 5 .

2. Lekha Bhausaheb Kachare, "Object sorting robot using image processing”, Internatinal Journal of Engineering Science and Computing, Volume 5,Issue 7 , July 2017

3. Aji Joy, "Object sorting arm based on color 
sensing",International Journal of Advanced Research in

Electrical,Electronics and Instrumenation Engineering Volume 3, Issue 3, March 2014.

4. Rahul Kumar, Sunil Lal, Sanjesh Kumar, Praneel Chand, "Object

detection and recognition for a pick and place robot”, IEEE Asia-Pacific

World Congress on Computer Science and Engineering,November

2014.

\section{AUTHORS PROFILE}

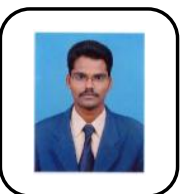

Dr. K.Vijayan has a total work experience of 16 years and 8 months. He completed his B.E in Electronics and Communication from Mahendra Engineering College, Salem, M.E in VLSI Design from College of Engineering, Guindy and $\mathrm{Ph} . \mathrm{D}$ in Electronics and Communication from Vels University, Chennai. He has published his work in 12 National and International Journals. He holds life memberships in professional societies like IACSIT, IAENG, ISTE,IETE and annual memberships in ISCA and IEEE. He is presently working as the Assistant Professor (Selection Grade) at SRM Institute of Science and Technology, Chennai, India.

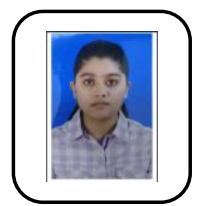

Anjima S Nair received her Bachelor's degree in Electronics and Communication from SRM Institute of Science and Technology, Chennai,India. She has done projects based on Weather monitoring system,water flow detector etc.

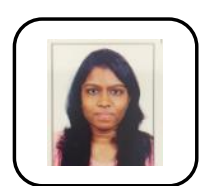

D Shruthi receieved her Bachelor's degree in Electronics and Communication from SRM Institute of Science and Technology, Chennai, India. Her major project works include real time railway crack detection, Home automation etc.

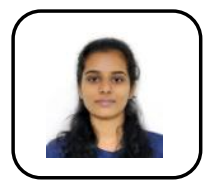

Krishna Manoj completed her Bachelor in Technology in Electronics and Communication from SRM Institute of Science and Technology, Chennai, India. Her major projects works include Weather monitoring system, projects based on MATLAB etc.

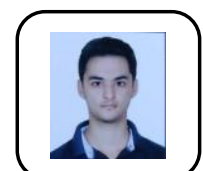

Rohan Sharma holds a Bachelor in Technology in Electronics and Communication from SRM Institute of Science and Technology, Chennai, India. His major project works include real time railway crack detection, Home automation etc. 